\title{
Patellar Height after High Tibial Osteotomy of the Distal Tibial Tuberosity: A Retrospective Study of Age Stratification
}

\author{
Tiansong Ding, ${ }^{1}$ Yetong Tan, ${ }^{2}$ Xiangdong Tian $\mathbb{D}^{2},{ }^{2}$ Zhipeng Xue, ${ }^{2}$ Sheng $\mathrm{Ma}^{2}$ Yuanyi Hu, ${ }^{2}$ \\ Ye Huang, ${ }^{1}$ and Xiaomin $\mathrm{Li}^{1}$ \\ ${ }^{1}$ Beijing University of Chinese Medicine, Beijing 100029, China \\ ${ }^{2}$ Minimal Invasive Joint Department, The Third Affiliated Hospital of Beijing University of Chinese Medicine, No. 51 Anwai \\ Xiaoguan Street, Chaoyang District, Beijing 100029, China
}

Correspondence should be addressed to Xiangdong Tian; c083@bucm.edu.cn

Received 7 November 2021; Revised 6 December 2021; Accepted 11 January 2022; Published 27 January 2022

Academic Editor: Kelvin Wong

Copyright ( 2022 Tiansong Ding et al. This is an open access article distributed under the Creative Commons Attribution License, which permits unrestricted use, distribution, and reproduction in any medium, provided the original work is properly cited.

\begin{abstract}
Objective. To explore the effect of age stratification on patellar height after single-plane high tibial osteotomy of the distal tibial tuberosity (DTT-HTO). Methods. A retrospective analysis was performed on 110 knee joints undergoing DTT-HTO. Patients were divided into three groups according to age: under 60 years old, 28 cases; 60 to 70 years old, 61 cases; and over 70 years old, 21 cases. All patients were followed up for no less than 12 months, and at each follow-up, short-leg radiographs and whole-leg radiographs were taken. The values of the Caton-Deschamps index (CDI) and Blackburne-Peel index (BPI) of single-short-leg radiographs and the femoral-tibial angle (FTA) and weight-bearing line ratio (WBLR) of whole-leg radiographs were measured before and at the last follow-up. The Lysholm score before and at the last follow-up and the visual analogue scale (VAS) score before and 3 days after surgery and at the last follow-up were calculated. The frequency of classification of the normal-height patella, patella alta, and patella baja before and after surgery was recorded. Results. There were no significant differences in CDI and BPI preoperatively or postoperatively among the three groups $(P>0.05)$, and there were no statistically significant differences in FTA and WBLR. There were no significant differences in CDI, BPI, FTA, or WBLR between the three groups before and after the operation $(P>0.05)$. The Lysholm score increased from $48.84 \pm 10.10$ before surgery to $91.96 \pm$ 3.082 after surgery $(P<0.05)$; the VAS score decreased from $8.23 \pm 0.99$ before surgery to $1.93 \pm 0.953$ at 3 days after surgery and $1.07 \pm 0.53$ at the last follow-up $(P<0.01)$. No significant difference was observed in the incidence of each patellar height classification between the three groups preoperatively and postoperatively. Conclusion. Patellar height is not influenced by DTT-HTO. The age of patients is not a limiting factor for the selection of this surgical procedure. Without affecting the height of the patella, DTT-HTO can effectively reduce pain in the knee joint, restore the function of the knee joint, and delay the progression of patellar arthritis.
\end{abstract}

\section{Introduction}

High tibial osteotomy is one of the surgical methods for treating medial compartment knee osteoarthritis. Traditional HTO is divided into closing-wedge high tibial osteotomy (CWHTO) and open-wedge high tibial osteotomy (OWHTO), and both procedures can effectively correct the coronal weight-bearing line of the knee joint, transfer the lower limb alignment from the medial compartment of the knee joint to the lateral compartment, increase the stress area of the tibiofemoral joint, and reduce the pressure of the medial compartment to relieve medial compartment knee pain and help patients recover walking function [1-13]. The mechanical properties of a biostructure depend on its Young's modulus $(E)$. Therefore, it is likely that a harder substance has a larger $E$ [14]. For instance, the value of $E$ for bone is $14 \mathrm{GPa}$. However, HTO can also affect the postoperative bone structure of the patellofemoral joint, resulting in patella alta or patella baja compared to the preoperative situation. Therefore, measurement methods [15] such as CDI [16] and BPI [16] are often used to test whether the height of the patella changes. 
TABLE 1: Characteristics of the three groups.

\begin{tabular}{|c|c|c|c|c|c|}
\hline & $<60$ years $(n=28)$ & $60 \sim 70$ years $(n=61)$ & $>70$ years $(n=21)$ & Value & $P$ \\
\hline \multicolumn{6}{|l|}{ Sex } \\
\hline Male & 8 & 15 & 5 & $\chi^{2}=0.197$ & 0.906 \\
\hline Female & 20 & 46 & 16 & & \\
\hline \multicolumn{6}{|l|}{ Knee } \\
\hline Left & 20 & 33 & 15 & $\chi^{2}=3.458$ & 0.178 \\
\hline Right & 8 & 28 & 6 & & \\
\hline Duration of disease (years) & $8.50 \pm 1.972$ & $8.85 \pm 2.056$ & $9.29 \pm 2.935$ & $F=0.747$ & 0.476 \\
\hline BMI (body mass index) $\left(\mathrm{kg} / \mathrm{m}^{2}\right)$ & $26.40 \pm 3.046$ & $26.38 \pm 3.195$ & $25.89 \pm 2.586$ & $F=0.224$ & 0.800 \\
\hline \multicolumn{6}{|l|}{ BMD $t$-score } \\
\hline$\leq-2.5(\%)$ & 2 & 9 & 6 & $\chi^{2}=5.508$ & 0.239 \\
\hline$>-2.5 \sim \leq-1.5(\%)$ & 5 & 15 & 6 & & \\
\hline$>-1.5(\%)$ & 19 & 37 & 9 & & \\
\hline Varus deformity $\left({ }^{\circ}\right)$ & $8.71 \pm 2.088$ & $9.16 \pm 2.099$ & $9.62 \pm 2.519$ & $F=1.043$ & 0.356 \\
\hline Duration of follow-up (months) & $20.86 \pm 5.707$ & $21.64 \pm 4.401$ & $24.05 \pm 4.78$ & $F=2.815$ & 0.064 \\
\hline
\end{tabular}

There are different opinions about the effect of HTO on patellar height, but a consensus has already been reached regarding whether high tibial osteotomy above the tibial tuberosity can affect patellar height $[6,12,17-20]$. This was confirmed by previous retrospective clinical studies of DTT-HTO conducted by our team [9]. Patellar height disorder can lead to cartilage degeneration of the patellofemoral joint, loss of function, knee pain, and recurrent patellofemoral dislocation. The pressure on the patellofemoral joint increases during the bending of the patella baja; therefore, the possibility of patellofemoral joint degeneration increases [2]. Patellar instability is often associated with patellar alta, and long-term patellar instability can lead to knee pain, functional limitations, and osteoarthritis. The inability causes the patella to move outward when knee flexural activity, especially during the first $30^{\circ}$, is unable to pull the patella [21]. Because of the patella alta state, the contact area between the patella and the femur decreases, the pressure increases, and the patellofemoral joint wear increases, resulting in patellofemoral chondromalacia [22].

Current literature reports that $\mathrm{HTO}$ is suitable for young, active knee osteoarthritis patients with high functional needs. In previous studies, 60 years [23-25] or 65 years [26] was the age limit often used as the inclusion criterion for HTO surgical indicators. This may be due to the influence of certain variables (such as osteoporosis) that change with age on the outcome of surgery. However, it has been found that a number of knee osteoarthritis (KOA) patients aged over 60 or 65 years old also achieve good clinical efficacy by HTO in clinical practice. They often experience relief from knee pain in a short period of time and gradually recover walking, going up and down the stairs, flexion and extension functions, and so on. More research on the effect of HTO on patellar height has focused on the choice of surgical procedures; some studies have challenged the age limitation in HTO inclusion criteria, but no literature has been found on the effect of age on patellar height after HTO. The purpose of this study was to observe the influence of age on patellar height after DTT-HTO and to explore whether the age restriction can be broken and whether more surgical options can be provided to patients with KOA.

\section{Methods}

2.1. Patients. A retrospective study of 128 patients who received DTT-HTO in the Third Affiliated Hospital of Beijing University of Chinese Medicine from August 2016 to June 2020 was conducted. Except for the 18 cases that were removed according to the inclusion and exclusion criteria, a total of 110 patients aged 51 to 82 were divided into three groups according to age: under 60 years old, 28 cases; 60 to 70 years old, 61 cases; and over 70 years old, 21 cases. There was no significant difference in demographics or parameters among the three groups (Table 1). Inclusion criteria are as follows: (1) medial compartment arthritis of grade $\mathrm{k}-\mathrm{L}$ : $\geq 3$, lateral: $\leq 1$; (2) follow-up time $\geq 12$ months; and (3) image data that were completed and that comprised approximately $30^{\circ}$ flexion knee lateral radiographs and whole-leg radiographs before and at the last follow-up. Exclusion criteria are as follows: (1) rotational angles existing between the medial femoral condyle and the lateral femoral condyle in one of the lateral radiographs and (2) patients suffering from types of arthritis other than KOA.

2.2. Operative Procedure. Starting from $1 \mathrm{~cm}$ below the midpoint of the medial joint line, an incision of approximately $5 \mathrm{~cm}$ was made downward to separate the skin, subcutaneous, deep fascia, muscle, and periosteum in turn to expose the tibial bone surface (Figure 1(a)), avoiding harming the patellar tendon and pes anserinus. A Kirschner wire was inserted from the distal tibial tubercle to $0 \sim 1 \mathrm{~cm}$ below the tibiofibular fornix at an angle of $30^{\circ}$ to $45^{\circ}$ to the vertical line of the longitudinal axis of the tibia. The position of the 


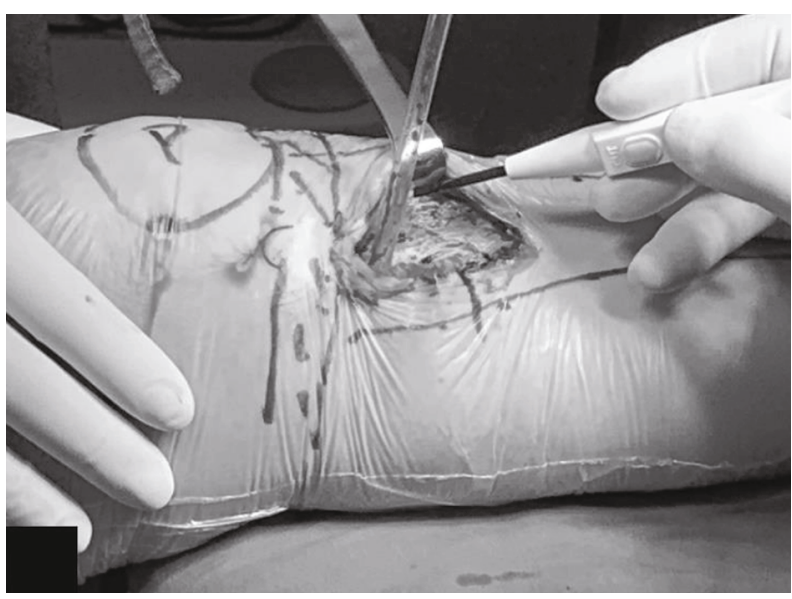

(a)

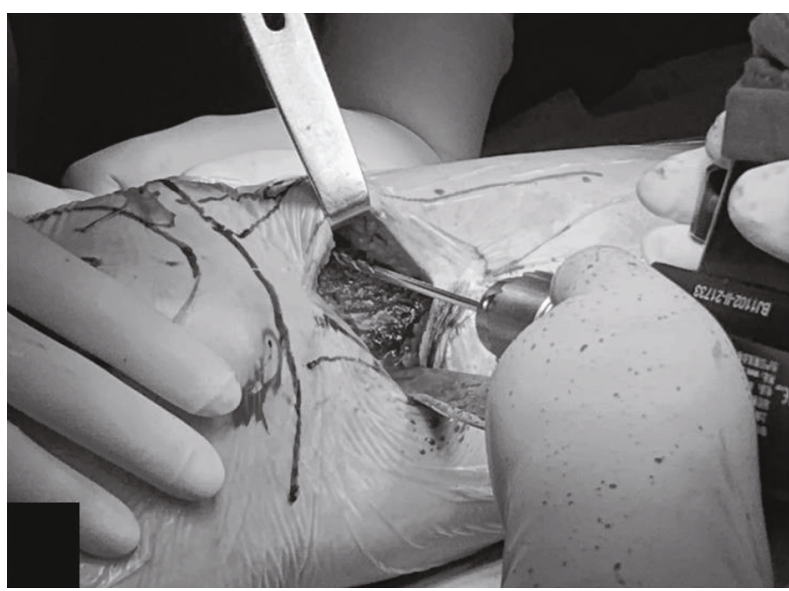

(c)

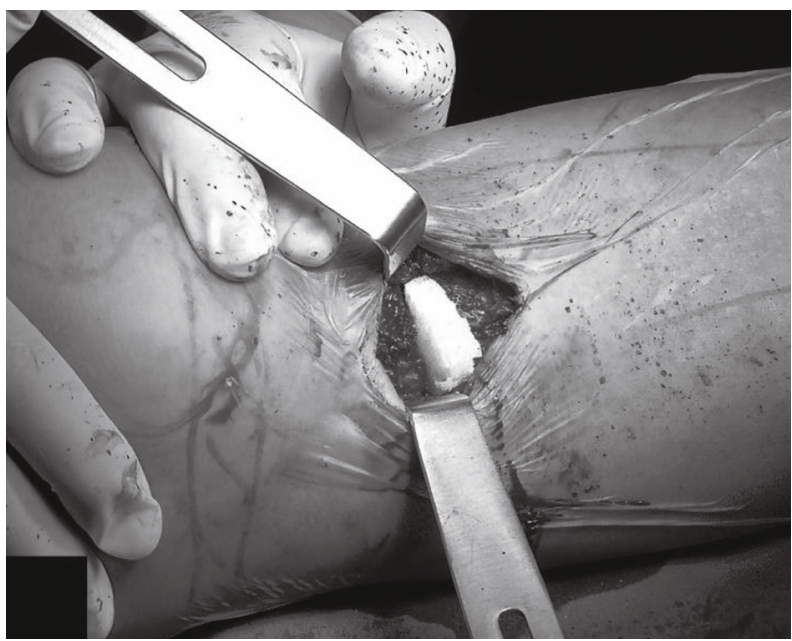

(e)

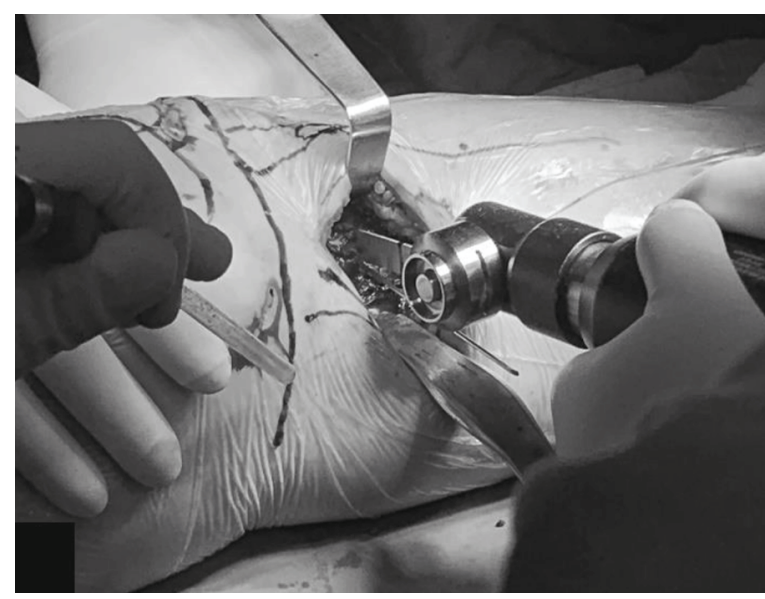

(b)

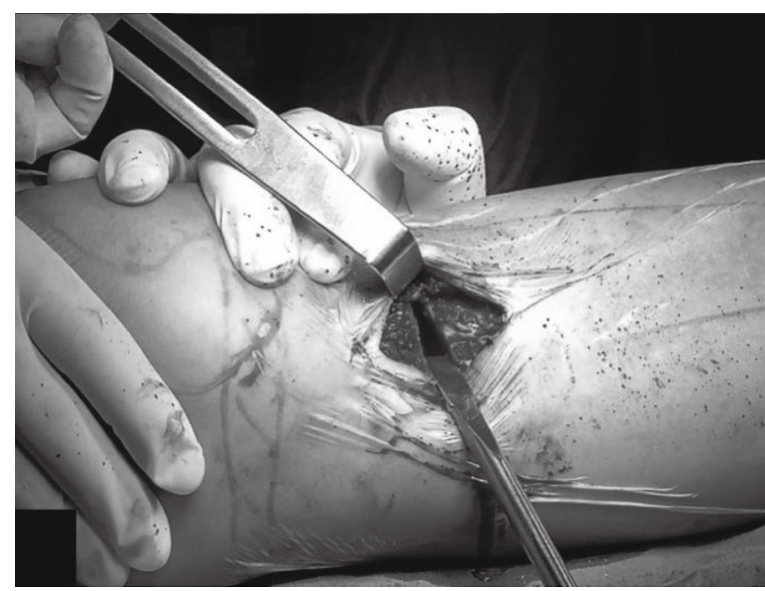

(d)

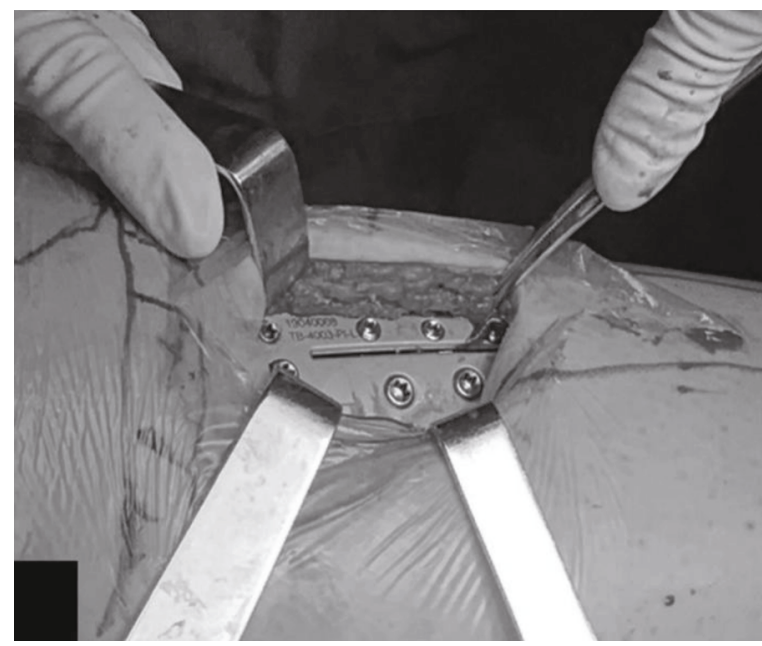

(f)

Figure 1: Intraoperative operation of DTT-HTO. (a) Exposure of the tibial bone surface. (b) The medial tibia was sawed by a bone saw along the Kirschner wire route. (c) Use of a Kirschner wire to drill holes from the osteotomy area to the opposite side to weaken the lateral bone cortex. (d) Opening up the osteotomy area with the bone knife. (e) Implantation of bone substitutes. (f) Placement of the guide plate and its fixation with screws.

Kirschner wire was observed on the C-arm, and a bone saw cut the medial tibia off along the Kirschner wire (Figure 1(b)), leaving $1 \mathrm{~cm}$ of cortical bone on the lateral side of the tibia. The lateral cortex was loosened by drilling holes from the osteotomy area to the lateral residual cortex (Figure 1(c)). The assistant performed valgus and rotation stress on the operative limb, and the surgeon used a bone knife to open it up (Figure $1(\mathrm{~d})$ ) to change the lower limb 


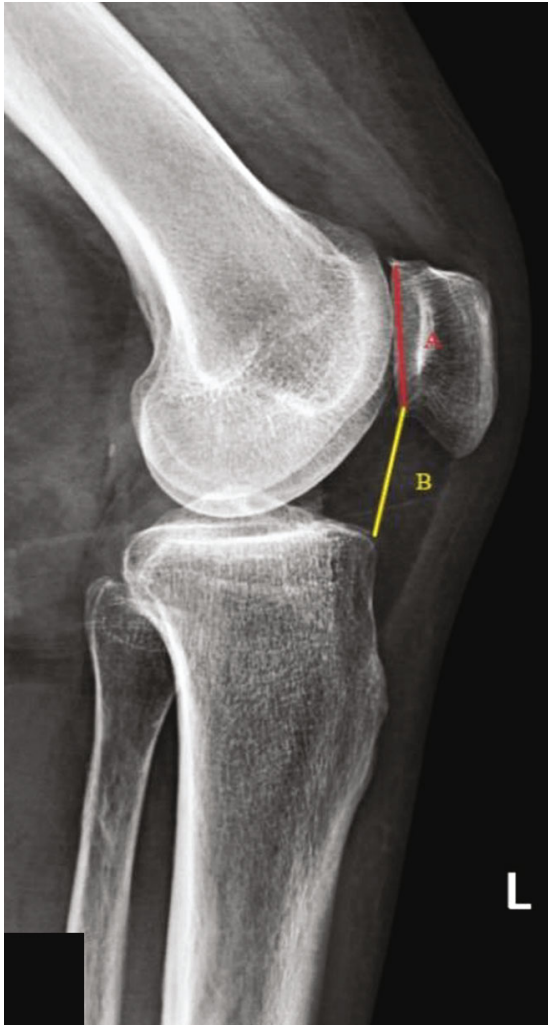

(a)

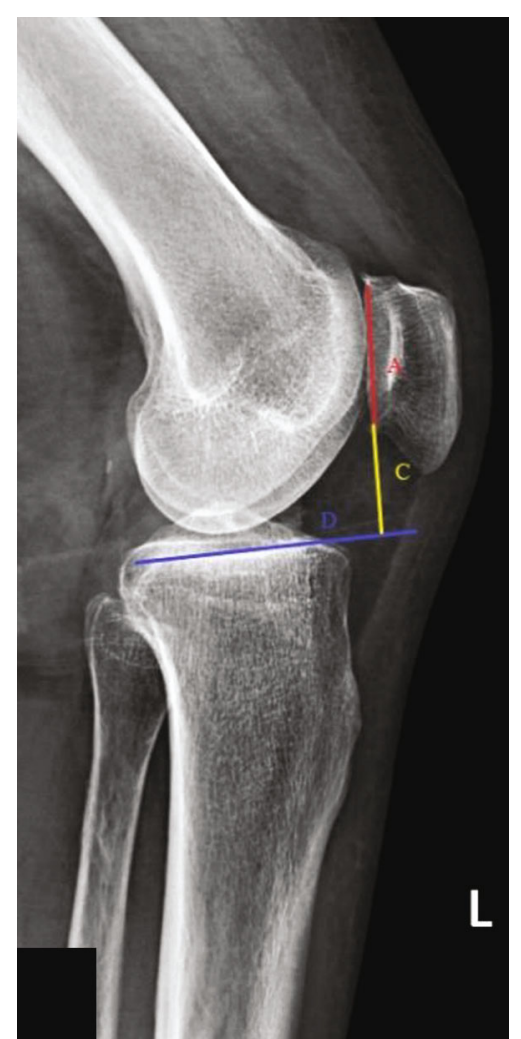

(b)

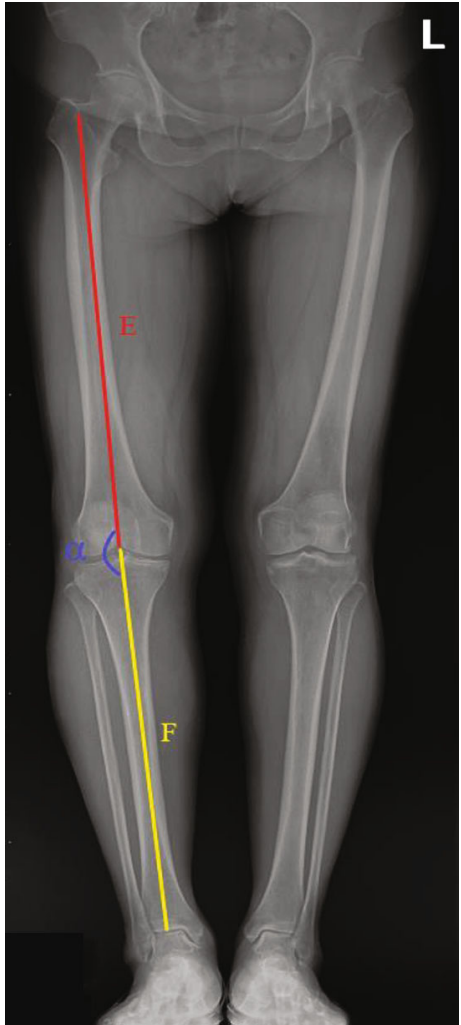

(c)

Figure 2: Methods of image measurement. (a) $\mathrm{CDI}=B / A$. (b) $\mathrm{BPI}=C / A$. (c) $\mathrm{FTA}=\alpha$.

alignment. C-arm fluoroscopy showed that the WBL passed lateral to the lateral tibial intercondylar eminence at the Fujisawa point. Bone substitutes were implanted (Figure 1(e)), a guide plate and screws were inserted (Figure 1(f)), and the wound was finally sewn up.

Ankle pump exercises were performed in bed on the first day after surgery, and patients were allowed to step on the ground with the aid of a walker on the third postoperative day. The patients advanced to walking with two crutches two weeks after the surgery and with a single crutch four weeks after the surgery. Walking without auxiliary devices was achieved 8 to 12 weeks after the surgery.

2.3. Radiological Measurements. All patients were photographed before surgery and at the last follow-up to observe the fixation of the guide plate and screws and the changes in CDI, BPI, FTA, and WBLR. All images were taken by the same technician and the same machine (GE Definium 6000 DR) and were independently measured by two project team members.

The Caton-Deschamps index is the ratio between the length from the lowest point of the patellar articular surface to the anterior tip of the tibial tuberosity and the patellar joint surface $(B / A)$. BPI is the ratio of the vertical distance from the lowest point of the patellar articular surface to the medial tibial plateau line $(D)$ to the patellar articular surface $(C / A)$. FTA is the angle $(\alpha)$ formed by the anatomical axis of the femur $(F)$ and tibia $(G)$ at the center of the knee joint (Figure 2). WBLR is the distance from the intersection of the lower limb alignment and the tibial plateau to the medial edge of the tibial plateau $(H)$ divided by the width of the tibial plateau (I) (Figure 3 ). The CDI and BPI were measured on lateral radiographs in mild degrees of flexion $\left(30^{\circ}\right)$. FTA and WBLR were gauged on non-weight-bearing lateral radiographs.

2.4. Clinical Evaluation. The Lysholm score was used to assess the knee status before and at the last follow-up, including gait, function, pain, swelling, and stability. The visual analogue scale (VAS) was used to assess the degree of pain before and 3 days after surgery and at the last follow-up, with a score ranging from 0 to 10 . The frequencies of the normal-height patella, high patella, and low patella in the three groups were compared before and after the operation.

2.5. Statistical Analysis. All the collected data were analyzed using SPSS (version 25.0; SPSS, China). A paired $t$-test was used to analyze the CDI, BPI, FTA, and WBLR in the groups. Single-sample analysis of variance was used between the groups. A paired $t$-test was used to compare the preoperative and postoperative Lysholm scores, and repeatedmeasure ANOVA was used to detect the changes in VAS scores before surgery, 3 days after surgery, and at the last follow-up. The chi-square test was used to compare the changes in patellar height classification in the three groups before and after surgery. 


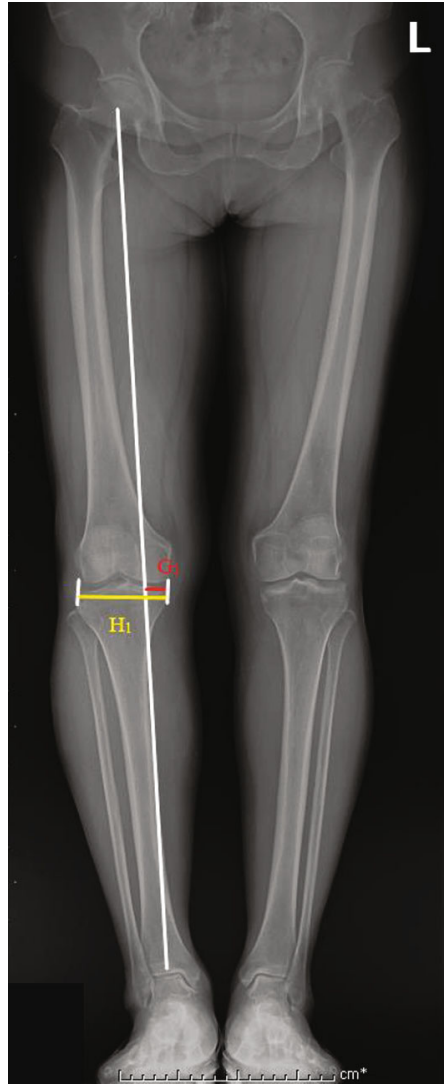

(a)

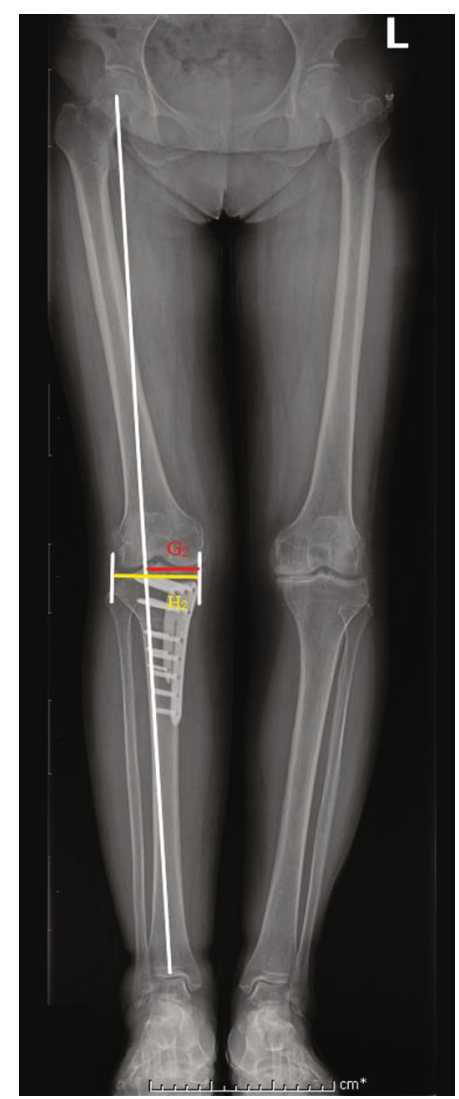

(b)

Figure 3: Method of WBL. (a) $\mathrm{WBLR}_{1}=H_{1} / I_{1}$. (b) $\mathrm{WBLR}_{2}=H_{2} / I_{2}$.

\section{Results}

There were no significant differences in CDI and BPI preoperatively or postoperatively among the three groups $(P>0.05)$, and the differences in FTA and WBLR were statistically significant $(P<0.05)$. No significant differences were observed in CDI, BPI, FTA, or WBLR between the three groups before and after surgery $(P>0.05)$ (Table 2$)$.

The Lysholm score of the knees increased from $48.84 \pm$ 10.10 before the operation to $91.96 \pm 3.082$ at the last follow-up $(P<0.05)$. The VAS score decreased from $8.23 \pm$ 0.99 before surgery to $1.93 \pm 0.953$ on the third postoperative day and $1.07 \pm 0.53$ at the last follow-up $(P<0.01)$ (Table 3$)$.

The incidences of the normal patella, patella alta, and patella baja (CDI $<0.8$ was taken as the standard) were $53.57 \%, 17.86 \%$, and $28.57 \%$, respectively, in the $<60$-yearold group before surgery and $50.00 \%, 17.86 \%$, and $32.14 \%$, respectively, at the last follow-up. The incidences of the three types of patellar heights in the 60 70-year-old group were $52.50 \%, 14.75 \%$, and $32.79 \%$, respectively, before surgery and $47.54 \%, 14.75 \%$, and $37.70 \%$, respectively, at the last followup. The incidences of the three types of patellar heights before surgery in the $>70$-year-old group were $52.50 \%, 14.75 \%$, and $32.79 \%$, and these incidences were $47.54 \%, 14.75 \%$, and $37.70 \%$, respectively, at the last follow-up. There were no significant differences among the three groups preoperatively and postoperatively (Table 4).
One patient in the $<60$-year-old group, five patients in the 60 70-year-old group, and one patient in the $>70$ year-old group had a suspicious low patella after knee surgery $(0.6<\mathrm{CDI}<0.8)$. Decreases in patellar height were not observed after surgery in the three groups $(\mathrm{CDI}<0.6)$ (Table 5).

\section{Discussion}

4.1. Influence of HTO on Patellar Height in Previous Literature. CWHTO has a long history. Disadvantages of bone mass on the tibiofibular joint and the risk of common fibular nerve injury have caused it to be surpassed by OWHTO, which can avoid these problems and control the osteotomy angle and WBL more accurately and easily; however, bone substitutes are needed, and the healing speed declines in the osteotomy area because of cortical bone [4]. The patellar tendon is the lower part of the quadriceps femoris muscle, starting from the lower margin of the patella and ending at the tuberosity of the tibia [27]. Classical OWHTO was performed through the superior tibial tuberosity. Expansion of the osteotomy area resulted in the distal displacement of the tibial tuberosity and increased tensile stress of the patellar tendon, which pulled the patella to the distal end of the limb and led to changes in the height of the patella [5]. On this basis, the greater the osteotomy angle, the more obvious the patellar height decrease [13]. 
TABLE 2: Changes in imaging indicators in the three groups $(\bar{x} \pm s)$.

\begin{tabular}{|c|c|c|c|c|c|}
\hline & Groups & Preoperative & Postoperative & $t$ & $P$ \\
\hline & $<60$ years & $0.88 \pm 0.11$ & $0.88 \pm 0.121$ & -0.113 & 0.911 \\
\hline \multirow[t]{2}{*}{ CDI } & $60 \sim 70$ years & $0.87 \pm 0.15$ & $0.85 \pm 0.156$ & 1.569 & 0.122 \\
\hline & $>70$ years & $0.90 \pm 0.132$ & $0.89 \pm 0.131$ & 1.333 & 0.197 \\
\hline$F$ & & 0.422 & 0.537 & & \\
\hline$P$ & & 0.657 & 0.586 & & \\
\hline & $<60$ years & $0.85 \pm 0.124$ & $0.87 \pm 0.126$ & -0.854 & 0.401 \\
\hline \multirow[t]{2}{*}{ BPI } & $60 \sim 70$ years & $0.89 \pm 0.128$ & $0.88 \pm 0.131$ & 1.986 & 0.052 \\
\hline & $>70$ years & $0.90 \pm 0.08$ & $0.91 \pm 0.071$ & -0.285 & 0.779 \\
\hline$F$ & & 1.256 & 0.812 & & \\
\hline$P$ & & 0.289 & 0.447 & & \\
\hline & $<60$ years & $0.18 \pm 0.084$ & $0.55 \pm 0.089$ & -23.184 & $<0.01$ \\
\hline \multirow[t]{2}{*}{ WBLR } & $60 \sim 70$ years & $0.18 \pm 0.138$ & $0.59 \pm 0.10$ & -20.136 & $<0.01$ \\
\hline & $>70$ years & $0.19 \pm 0.089$ & $0.61 \pm 0.041$ & -19.481 & $<0.01$ \\
\hline$F$ & & 0.029 & 0.713 & & \\
\hline$P$ & & 0.972 & 0.492 & & \\
\hline & $<60$ years & $182.96 \pm 2.063$ & $172.39 \pm 3.919$ & 15.884 & $<0.01$ \\
\hline \multirow[t]{2}{*}{ FTA } & $60 \sim 70$ years & $183.57 \pm 2.171$ & $171.02 \pm 3.359$ & 25.800 & $<0.01$ \\
\hline & $>70$ years & $183.48 \pm 2.112$ & $170.62 \pm 2.459$ & 18.605 & $<0.01$ \\
\hline$F$ & & 0.830 & 2.046 & & \\
\hline$P$ & & 0.439 & 0.134 & & \\
\hline
\end{tabular}

TABle 3: Preoperative and postoperative score changes of the affected knee $(\bar{x} \pm s)$.

\begin{tabular}{lccccc}
\hline & Preoperative & Third postoperative day & Last follow-up & Value & $P$ \\
\hline Lysholm & $48.84 \pm 10.10$ & - & $91.96 \pm 3.082$ & $t=-36.662$ & $<0.01$ \\
VAS & $8.23 \pm 0.99$ & $1.93 \pm 0.953$ & $1.07 \pm 0.53$ & $F=1461.907$ & $<0.01$ \\
\hline
\end{tabular}

TABLE 4: Changes in the frequency of patellar height classification $(n=110, \%)$.

\begin{tabular}{|c|c|c|c|c|c|c|}
\hline & & Normal patella & Patella alta & Patella baja & $\chi^{2}$ & $P$ \\
\hline \multirow{2}{*}{$<60$ years } & Preoperative & $15(53.57)$ & $5(17.86)$ & $8(28.57)$ & \multirow{2}{*}{0.093} & \multirow{2}{*}{0.954} \\
\hline & Postoperative & $14(50.00)$ & $5(17.86)$ & $9(32.14)$ & & \\
\hline \multirow{2}{*}{$60 \sim 70$ years } & Preoperative & $32(52.50)$ & $9(14.75)$ & $20(32.79)$ & \multirow{2}{*}{0.357} & \multirow{2}{*}{0.837} \\
\hline & Postoperative & $29(47.54)$ & $9(14.75)$ & $23(37.70)$ & & \\
\hline \multirow{2}{*}{$>70$ years } & Preoperative & $11(52.38)$ & $5(23.81)$ & $5(23.81)$ & \multirow{2}{*}{0.155} & \multirow{2}{*}{0.926} \\
\hline & Postoperative & $12(57.14)$ & $5(23.81)$ & $4(19.05)$ & & \\
\hline
\end{tabular}

Contracture of the patellar tendon after osteotomy may also result in a decrease in the height of the patella [28].

CWHTO avoids osteotomy above the tibial tuberosity. In the meta-analysis of 28 trials included by Cheng et al. [29], no significant change in patellar height was seen after CWHTO. Tigani et al. [6] measured the patellar height after OWHTO and CWHTO by CDI and found that the patellar height of OWHTO decreased more significantly during at least 1 year of postoperative follow-up. Even in OWHTO, biplanar descending OWHTO (osteotomy line at the distal tibial tuberosity) did not affect patellar height compared with biplanar ascending OWHTO (osteotomy line at the proximal tibial tuberosity) [7]. Warner et al. [5] performed subtubercle osteotomy in the treatment of MCOA combined with the Ilizarov technique. They found that the height of the patella (CDI) in 72 knees did not change significantly after the operation and that the patellofemoral relationship was normal. Next, Bayam et al. [8] combined STO with the fixator-assisted nailing technique to perform orthopedic procedures on 32 knee joints, and the study confirmed that the patellar height was not affected during at least 30 months of follow-up. In a large number of clinical studies, it has been 
TABLE 5: Outcomes of patellar height in the three groups $(n=110$, cases).

\begin{tabular}{|c|c|c|c|}
\hline & Preoperative & \multicolumn{2}{|c|}{ Postoperative } \\
\hline \multirow{12}{*}{$<60$ years } & \multirow{2}{*}{ Normal patella } & \multicolumn{2}{|c|}{ Normal patella } \\
\hline & & + & - \\
\hline & + & 14 & 1 \\
\hline & - & 0 & 13 \\
\hline & \multirow{2}{*}{ Patella alta } & \multicolumn{2}{|c|}{ Patella alta } \\
\hline & & & - \\
\hline & + & 5 & 0 \\
\hline & - & 0 & 23 \\
\hline & \multirow{2}{*}{ Patella baja } & \multicolumn{2}{|c|}{ Patella baja } \\
\hline & & + & - \\
\hline & + & 8 & 0 \\
\hline & - & 1 & 19 \\
\hline \multirow{12}{*}{$60 \sim 70$ years } & \multirow{2}{*}{ Normal patella } & \multicolumn{2}{|c|}{ Normal patella } \\
\hline & & + & - \\
\hline & + & 27 & 5 \\
\hline & - & 2 & 27 \\
\hline & \multirow{2}{*}{ Patella alta } & \multicolumn{2}{|c|}{ Patella alta } \\
\hline & & + & - \\
\hline & + & 9 & 0 \\
\hline & - & 0 & 52 \\
\hline & \multirow{2}{*}{ Patella baja } & \multicolumn{2}{|c|}{ Patella baja } \\
\hline & & + & - \\
\hline & + & 18 & 2 \\
\hline & - & 5 & 36 \\
\hline \multirow{12}{*}{$>70$ years } & \multirow{2}{*}{ Normal patella } & \multicolumn{2}{|c|}{ Normal patella } \\
\hline & & + & - \\
\hline & + & 10 & 1 \\
\hline & - & 2 & 8 \\
\hline & \multirow{2}{*}{ Patella alta } & \multicolumn{2}{|c|}{ Patella alta } \\
\hline & & + & - \\
\hline & + & 5 & 0 \\
\hline & - & 0 & 16 \\
\hline & \multirow{2}{*}{ Patella baja } & \multicolumn{2}{|c|}{ Patella baja } \\
\hline & & + & - \\
\hline & + & 3 & 2 \\
\hline & - & 1 & 15 \\
\hline
\end{tabular}

confirmed that whether the osteotomy line passes through the tibial tuberosity is one of the evaluation criteria for the change in patellar height, and subtubercle osteotomy can effectively avoid this [3-5, 7-12].

Some studies [2] have shown that open-wedge proximal tibial osteotomy can significantly increase the pressure on the patellofemoral articular cartilage at $30^{\circ}, 60^{\circ}$, and $90^{\circ} \mathrm{knee}$ flexion and accelerate the degeneration of the patellofemoral articular cartilage. Sim et al. [30] found that patellar height decreased after OWHTO, and single-photon emission computed tomography and conventional computed tomography (SPECT/CT) were used to capture the contact stress of the patellofemoral joint. The results showed that the contact stress increased in $7.1 \%$ of the knees, the articular cartilage of the patella and femoral trochlea significantly deteriorated, and the grade of patellofemoral arthritis was elevated.

\subsection{Influence of Age on Radiological and Clinical Outcomes} after HTO. Although some studies on HTO have included age as a criterion, it should not be used as a mandatory screening criterion for the treatment of older KOA patients. One study found that radiological outcomes in patients receiving OWHTO were not affected by age, and clinical outcomes (HSS, KSS) were affected by cartilage status rather than age per se [31]. Kuwashima et al. [32] found that, compared with the preoperative KSS score, only the functional activity score showed differences in the group aged $\geq 65$ years and $\leq 64$ years, while differences in the symptom score, satisfaction score, and expectation score were not statistically significant. Kohn et al. [33] found that the improvement of pain symptoms (VAS score) and functional status (Lysholm score) of knees after HTO showed no significant difference between the young group and the old group. They believed that the postoperative efficacy of HTO patients was determined not by their age but by the state of knee soft tissue. Goshima et al. [34] divided OWHTO patients into two groups, older than 65 years old and younger than 65 years old, and conducted follow-up for up to 24 months. It was concluded that age did not affect the clinical and imaging results after HTO. To enhance the medical image analysis, superresolution image enhancement of the medical scans may be applied based on techniques such as deep learning [35].

4.3. The Effect of DTT-HTO on Patellar Height in This Study. DTT-HTO was simple to perform and effectively avoided patellar lowering or elevation. Seven of 110 knee joints decreased from the normal-height patella $(0.8<\mathrm{CDI}<1.2)$ to the low patella $(\mathrm{CDI}<0.8)$ in the image measurement data, but the overall height of the patella was not affected $(P>0.05)$, which may be related to measurement errors. The authors reviewed the factors affecting patellar height, including knee function, scarring on the patellar ligament, contracture [28], and the motion of the quadriceps femoris $[12,36]$. Therefore, preoperative protection of the knee joint, avoidance of accidental injury of the patellar ligament during the operation, and postoperative rehabilitation exercise can contribute to the recovery of knee joint function. Other various algorithms can be used to improve medical imaging procedures [37]. The bone structure integrity is heavily influenced by the material properties that are dependent on certain characteristics $[38,39]$.

4.4. Relationship of Patellar Height to Osteoporosis. Aging is one of the predisposing factors of osteoporosis; current international literature mainly considers the group aged $>60$ or 65 years as the high-risk population of osteoporosis [40]. Other operations of HTO, such as OWHTO, performed osteotomy above the tibial tuberosity, where there is abundant cancellous bone. Affected by the position of the osteotomy line, the supporting stress of internal fixation 
was mainly concentrated above the tibial tuberosity. If the patient with KOA also has osteoporosis, the screws, inserted to fix the guide, have the potential to cut cancellous bone under body gravity, resulting in bone loss and biomechanical environment disorder in the patellofemoral joint. DTTHTO can avoid this happening effectively.

4.5. Limitation. The limitations of our study are as follows. (1) The short follow-up time made it impossible to explore the long-term effect of DTT-HTO on patellar height. (2) The observation indicators mainly focused on the image measurement method, and the results had errors. (3) Other factors contributing to the changes in body mechanisms associated with aging have not been explored more broadly. (4) The influence of osteoporosis on HTO has not been further studied.

\section{Conclusion}

DTT-HTO did not change the height of the patella at different ages, WBL and FTA were corrected, pain was relieved, and function was recovered. Based on the clinical trial results of this study, patients should not be forced to give up their choice of HTO simply because of their age. We recommend that the inclusion criteria for HTO be improved to provide more surgical options and benefit elderly KOA patients.

\section{Abbreviations}

$\begin{array}{ll}\text { KOA: } & \text { Knee osteoarthritis } \\ \text { DTT-HTO: } & \text { Distal tibial tuberosity high tibial osteotomy } \\ \text { CDI: } & \text { Caton-Deschamps index } \\ \text { BPI: } & \text { Blackburne-Peel index } \\ \text { VAS: } & \text { Visual analogue scale } \\ \text { FTA: } & \text { Femoral-tibial angle } \\ \text { WBLR: } & \text { Weight-bearing line ratio } \\ \text { CWHTO: } & \text { Closing-wedge high tibial osteotomy } \\ \text { OWHTO: } & \text { Open-wedge high tibial osteotomy. }\end{array}$

\section{Data Availability}

Data are available on request from the authors due to privacy/ethical restrictions.

\section{Ethical Approval}

All human subjects in this study have given their written consent for participation in our research.

\section{Consent}

Signed informed consent for publication was obtained from all authors.

\section{Conflicts of Interest}

The authors declare no conflict of interest.

\section{Authors' Contributions}

XDT designed the study. TSD collected the data and wrote the manuscript. YTT, SM, YYH, and ZPX performed the surgery. YH and XML collected the data and discussed the results. All authors read and approved the final manuscript.

\section{Acknowledgments}

The authors thank all members of the Third Affiliated Hospital of Beijing University of Chinese Medicine who contributed to this research. This study was funded by the Third Affiliated Hospital of Beijing University of Chinese Medicine (No. BZYSY-QNJS-2019-JYB-JS-178).

\section{References}

[1] T. O. Smith, D. Sexton, P. Mitchell, and C. B. Hing, "Openingor closing-wedged high tibial osteotomy: a meta-analysis of clinical and radiological outcomes," The Knee, vol. 18, no. 6, pp. 361-368, 2011.

[2] K. Stoffel, C. Willers, O. Korshid, and M. Kuster, "Patellofemoral contact pressure following high tibial osteotomy: a cadaveric study," Knee Surgery, Sports Traumatology, Arthroscopy, vol. 15, no. 9, pp. 1094-1100, 2007.

[3] S. G. Gooi, C. X. Y. Chan, M. K. L. Tan, A. K. S. Lim, K. Satkunanantham, and J. H. P. Hui, "Patella height changes post high tibial osteotomy," Indian J Orthop, vol. 51, no. 5, pp. 545-551, 2017.

[4] J. S. Shim, S. H. Lee, H. J. Jung, and H. I. Lee, "High tibial open wedge osteotomy below the tibial tubercle: clinical and radiographic results," Knee Surgery, Sports Traumatology, Arthroscopy, vol. 21, no. 1, pp. 57-63, 2013.

[5] S. J. Warner, D. P. O'Connor, and M. R. Brinker, "Subtubercle osteotomy for medial compartment osteoarthritis of the knee using Ilizarov technique: survival analysis and clinical outcomes," The Journal of Bone and Joint Surgery. American Volume, vol. 100, no. 1, 2018.

[6] D. Tigani, D. Ferrari, P. Trentani, G. Barbanti-Brodano, and F. Trentani, "Patellar height after high tibial osteotomy," International Orthopaedics, vol. 24, no. 6, pp. 331-334, 2001.

[7] M. Krause, T. C. Drenck, A. Korthaus, A. Preiss, K. H. Frosch, and R. Akoto, "Patella height is not altered by descending medial open-wedge high tibial osteotomy (HTO) compared to ascending HTO," Knee Surgery, Sports Traumatology, Arthroscopy, vol. 26, no. 6, pp. 1859-1866, 2018.

[8] L. Bayam, M. Erdem, D. Gulabi, A. C. Erdem, A. C. Uyar, and A. Kochai, "Clinical and radiological outcomes of high tibial osteotomy with combined fixator-assisted nailing and subtubercle tibial osteotomy," Acta Orthopaedica et Traumatologica Turcica, vol. 54, no. 1, pp. 89-96, 2020.

[9] C. Han, X. Li, X. Tian et al., "The effect of distal tibial tuberosity high tibial osteotomy on postoperative patellar height and patellofemoral joint degeneration," Journal of Orthopaedic Surgery and Research, vol. 15, no. 1, p. 466, 2020.

[10] R. D. A. Gaasbeek, H. Sonneveld, R. J. van Heerwaarden, W. C. H. Jacobs, and A. B. Wymenga, "Distal tuberosity osteotomy in open wedge high tibial osteotomy can prevent patella infera: a new technique," The Knee, vol. 11, no. 6, pp. 457-461, 2004.

[11] P. D. Longino, T. B. Birmingham, W. J. Schultz, R. F. Moyer, and J. R. Giffin, "Combined tibial tubercle osteotomy with 
medial opening wedge high tibial osteotomy minimizes changes in patellar height: a prospective cohort study with historical controls," The American Journal of Sports Medicine, vol. 41, no. 12, pp. 2849-2857, 2013.

[12] H. El-Azab, P. Glabgly, and J. Paul, "Patellar height and posterior tibial slope after open- and closed-wedge high tibial osteotomy: a radiological study on 100 patients," The American Journal of Sports Medicine, vol. 38, no. 2, pp. 323-329, 2010.

[13] R. Gaasbeek, R. Welsing, M. Barink, N. Verdonschot, and A. van Kampen, "The influence of open and closed high tibial osteotomy on dynamic patellar tracking: a biomechanical study," Knee Surgery, Sports Traumatology, Arthroscopy, vol. 15, no. 8, pp. 978-984, 2007.

[14] K. K. L. Wong, "Three-dimensional discrete element method for the prediction of protoplasmic seepage through membrane in a biological cell," Journal of Biomechanics, vol. 65, no. 65, pp. 115-124, 2017.

[15] L. Sun, Q. Kong, Y. Huang et al., “Automatic segmentation and measurement on knee computerized tomography images for patellar dislocation diagnosis," Computational and Mathematical Methods in Medicine, vol. 2020, Article ID 1782531, 2020.

[16] C. Ihle, M. Ahrend, L. Grünwald, A. Ateschrang, U. Stöckle, and S. Schröter, "No change in patellar height following open wedge high tibial osteotomy using a novel femur-referenced measurement method," The Knee, vol. 24, no. 5, pp. 11181128, 2017.

[17] J. Amzallag, N. Pujol, A. Maqdes, P. Beaufils, T. Judet, and Y. Catonne, "Patellar height modification after high tibial osteotomy by either medial opening-wedge or lateral closingwedge osteotomies," Knee Surgery, Sports Traumatology, Arthroscopy, vol. 21, no. 1, pp. 255-259, 2013.

[18] D. Qin, W. Chen, J. Wang et al., "Mechanism and influencing factors of proximal fibular osteotomy for treatment of medial compartment knee osteoarthritis: a prospective study," The Journal of International Medical Research, vol. 46, no. 8, pp. 3114-3123, 2018.

[19] S. I. Bin, H. J. Kim, H. S. Ahn, D. S. Rim, and D. H. Lee, "Changes in patellar height after opening wedge and closing wedge high tibial osteotomy: a meta-analysis," Arthroscopy, vol. 32, no. 11, pp. 2393-2400, 2016.

[20] R. F. LaPrade, F. B. Oro, and C. G. Ziegler, "Patellar height and tibial slope after opening-wedge proximal tibial osteotomy: a prospective study," The American Journal of Sports Medicine, vol. 38, no. 1, pp. 160-170, 2010.

[21] N. A. Watson, K. R. Duchman, N. M. Grosland, and M. J. Bollier, "Finite element analysis of patella alta: a patellofemoral instability model," The Iowa Orthopaedic Journal, vol. 37, pp. 101-108, 2017.

[22] K. K. Middleton, S. Gruber, and B. E. Shubin Stein, "Why and where to move the tibial tubercle: indications and techniques for tibial tubercle osteotomy," Sports Medicine and Arthroscopy Review, vol. 27, no. 4, pp. 154-160, 2019.

[23] G. Papachristou, S. Plessas, J. Sourlas, C. Levidiotis, E. Chronopoulos, and C. Papachristou, "Deterioration of long-term results following high tibial osteotomy in patients under 60 years of age," International Orthopaedics, vol. 30, no. 5, pp. 403-408, 2006.

[24] I. J. Koh, M. S. Kim, S. Sohn et al., "Predictive factors for satisfaction after contemporary unicompartmental knee arthroplasty and high tibial osteotomy in isolated medial femorotibial osteoarthritis," Orthopaedics \& Traumatology, Surgery \& Research, vol. 105, no. 1, pp. 77-83, 2019.

[25] C. Bastard, G. Mirouse, D. Potage et al., "Return to sports and quality of life after high tibial osteotomy in patients under 60 years of age," Orthopaedics \& Traumatology, Surgery \& Research, vol. 103, no. 8, pp. 1189-1191, 2017.

[26] K. Trieb, J. Grohs, B. Hanslik-Schnabel, T. Stulnig, J. Panotopoulos, and A. Wanivenhaus, "Age predicts outcome of high-tibial osteotomy," Knee Surgery, Sports Traumatology, Arthroscopy, vol. 14, no. 2, pp. 149-152, 2006.

[27] M. Kazemi, Y. Dabiri, and L. P. Li, "Recent advances in computational mechanics of the human knee joint," Computational and Mathematical Methods in Medicine, vol. 2013, Article ID 718423, 27 pages, 2013.

[28] J. M. Wright, B. Heavrin, M. Begg, G. Sakyrd, and W. Sterett, "Observations on patellar height following opening wedge proximal tibial osteotomy," The American Journal of Knee Surgery, vol. 14, no. 3, pp. 163-173, 2001.

[29] X. Cheng, F. Liu, F. Xiong, Y. Huang, and A. C. Paulus, "Radiographic changes and clinical outcomes after open and closed wedge high tibial osteotomy: a systematic review and metaanalysis," Journal of Orthopaedic Surgery and Research, vol. 14, no. 1, p. 179, 2019.

[30] J. A. Sim, Y. G. Na, B. K. Lee, and B. H. Lee, “Alignment changes after open-wedge high tibial osteotomy result in offloading in the patellofemoral joint: a SPECT/CT analysis," Knee Surgery, Sports Traumatology, Arthroscopy, 2020.

[31] J. H. Song, S. I. Bin, J. M. Kim, and B. S. Lee, "Cartilage status, rather than chronologic age, determines the outcomes of open wedge high tibial osteotomy: a cartilage status-matched cohort study," Arthroscopy, vol. 37, no. 9, pp. 2915-2922, 2021.

[32] U. Kuwashima, K. Okazaki, K. Iwasaki et al., "Patient reported outcomes after high tibial osteotomy show comparable results at different ages in the mid-term to long-term follow-up," Journal of Orthopaedic Science, vol. 24, no. 5, pp. 855-860, 2019.

[33] L. Kohn, M. Sauerschnig, S. Iskansar et al., "Age does not influence the clinical outcome after high tibial osteotomy," Knee Surgery, Sports Traumatology, Arthroscopy, vol. 21, no. 1, pp. 146-151, 2013.

[34] K. Goshima, T. Sawaguchi, D. Sakagoshi, K. Shigemoto, Y. Hatsuchi, and M. Akahane, "Age does not affect the clinical and radiological outcomes after open-wedge high tibial osteotomy," Knee Surgery, Sports Traumatology, Arthroscopy, vol. 25, no. 3, pp. 918-923, 2017.

[35] M. Zhao, X. Liu, H. Liu, and K. Wong, "Super-resolution of cardiac magnetic resonance images using Laplacian pyramid based on generative adversarial networks," Computerized Medical Imaging and Graphics, vol. 80, p. 101698, 2020.

[36] K. W. Nha, A. Dorj, J. Feng et al., "Application of computational lower extremity model to investigate different muscle activities and joint force patterns in knee osteoarthritis patients during walking," Computational and Mathematical Methods in Medicine, vol. 2013, Article ID 314280, 9 pages, 2013.

[37] Z. H. Tang, Y. Y. Li, X. Y. Chai, H. Zang, and S. Cao, “Adaptive nonlinear model predictive control of NOx emissions under load constraints in power plant boilers," Journal of Chemical Engineering of Japan, vol. 53, no. 1, pp. 36-44, 2020.

[38] X. Xinli, C. Zhang, F. Musharavati, M. Demiral, A. M. Zain, and A. Khan, "UFSW tool pin profile effects on properties of 
aluminium-steel joint," Vacuum, vol. 192, no. 8, article 110460, 2021.

[39] X. Xinli, C. Zhang, and H. A. Derazkola, "Dispersion of waves characteristics of laminated composite nanoplate," Steel and Composite Structures, vol. 40, no. 3, pp. 355-367, 2021.

[40] I. R. Reid, "A broader strategy for osteoporosis interventions," Nature Reviews. Endocrinology, vol. 16, no. 6, pp. 333-339, 2020. 